\title{
Terapia dei prolattinomi: quando sospendere?
}

\author{
Renata S. Auriemma ${ }^{1}$. Roberta Scairati ${ }^{1}$. Guendalina del Vecchio ${ }^{1} \cdot$ Annamaria Colao $^{1,2}$
}

Accettato: 7 gennaio 2021 / Pubblicato online: 18 agosto 2021

(c) The Author(s) 2021

Sommario La gestione clinico-terapeutica dei pazienti con prolattinoma è basata sul trattamento con dopamino-agonisti (DA), in particolare la cabergolina, data la loro comprovata efficacia biochimica e antitumorale. Il raggiungimento di normali valori di prolattina associato alla significativa riduzione delle dimensioni tumorali, fino alla sua completa scomparsa, può suggerire l'opportunità di sospendere tale terapia nei pazienti trattati per almeno due anni. Tuttavia, è fortemente raccomandato di evitare la sospensione improvvisa del trattamento con DA e di preferire un protocollo di graduale riduzione del dosaggio di DA fino alla definitiva interruzione del trattamento, al fine di consentire la persistenza di normali valori di prolattina nel tempo. Nei pazienti con evidenza di recidiva di iperprolattinemia dopo un primo tentativo di sospensione dei DA, è possibile effettuare un secondo tentativo di sospensione dopo un ulteriore ciclo di trattamento con DA per due anni.

Parole chiave Prolattinoma - Dopamino-agonisti . Cabergolina $\cdot$ Sospensione del trattamento $\cdot$ Recidiva

Proposto da Massimo Mannelli, Francesco Ferraù, Lino Malandrino.

Informazioni Supplementari La versione online contiene materiale supplementare disponibile su

https://doi.org/10.1007/s40619-021-00929-x.

$凶$ R.S. Auriemma

renatasimona.auriemma@unina.it

1 Dipartimento di Medicina Clinica e Chirurgia, Università "Federico II", Napoli, Italia

2 Cattedra Unesco di Educazione alla Salute ed allo Sviluppo Sostenibile, Università "Federico II", Napoli, Italia

\section{Introduzione}

Il prolattinoma è un tumore benigno dell'adenoipofisi che determina un'eccessiva produzione di PRL, clinicamente caratterizzato da oligomenorrea, galattorrea, ipogonadismo e disfunzione sessuale, infertilità o, talora, asintomatico [1]. Come suggerito dalle linee guida internazionali della Endocrine Society del 2011 [1], il trattamento di prima scelta dei pazienti con prolattinoma è di tipo farmacologico ed è basato sulla somministrazione della terapia con dopaminoagonisti (DA), quali cabergolina (CAB) e bromocriptina (BRC). In particolare, le linee guida raccomandano l'utilizzo della $\mathrm{CAB}$ rispetto agli altri DA per la sua maggiore efficacia nel normalizzare i livelli di PRL, nel ridurre le dimensioni del tumore ipofisario e nel determinare il ripristino della funzione gonadica, con regressione della sintomatologia correlata all'eccesso di PRL e all'effetto massa [1]. Inoltre, in alcuni casi la terapia con DA è in grado di determinare la completa guarigione dei pazienti con prolattinoma e può, pertanto, essere sospesa in via definitiva [1-3]. Infatti, studi in vitro hanno dimostrato che un trattamento short-term (4-6 settimane) con DA è in grado di produrre la riduzione del citoplasma e delle aree nucleari e nucleolari, espressione di un globale effetto cistostatico $[4,5]$. Al contrario, una terapia long-term (14-36 settimane) con DA determina fibrosi perivascolare, degenerazione e necrosi, espressione di un globale effetto citotossico [6, 7]. Tuttavia, dagli studi attualmente disponibili emergono dati tuttora controversi riguardanti le modalità e il timing di sospensione del trattamento farmacologico.

\section{Quando sospendere la terapia?}

La sospensione del trattamento con DA è ancora oggi molto dibattuta e oggetto di grande attenzione da parte della co- 
munità scientifica. Il tasso di persistenti valori normali di prolattinemia dopo sospensione della terapia con DA varia sensibilmente tra gli studi a seconda del DA utilizzato e della durata del trattamento farmacologico prima della sospensione. D'altro canto, gli studi in letteratura hanno documentato in maniera concorde un rischio relativamente basso di ricrescita/recidiva tumorale nei primi mesi dopo la sospensione del trattamento con DA $[8,9]$.

\section{Bromocriptina}

Gli effetti della sospensione del trattamento con BRC sono stati approfonditamente valutati nel 2002 nello studio di Passos e collaboratori [10], che ha retrospettivamente analizzato la persistenza di normoprolattinemia dopo la sospensione di BRC in 350 pazienti con prolattinoma, di cui 189 con microprolattinoma e 169 con macroprolattinoma. I risultati di questo studio hanno dimostrato che solo nel 7,7\% dei pazienti si osservava persistenza di normali valori di PRL per un follow up medio di circa 44 mesi dopo sospensione di BRC [10]. Nessuno dei fattori valutati come possibili predittori di remissione di malattia, quali le dimensioni tumorali, i valori di PRL pretrattamento, il precedente intervento neurochirurgico di adenomectomia e la dose iniziale di BRC utilizzata, risultava significativamente associato alla persistenza di normoprolattinemia dopo sospensione della BRC [10]. Altri studi hanno riportato un tasso di remissione definitiva di malattia dopo sospensione del trattamento con BRC variabile dal 7 al 44\% [11-16], con evidenza biochimica di recidiva di malattia generalmente dopo 2-3 mesi dalla sospensione. Tuttavia, gli studi non hanno dimostrato un aumentato rischio di ri-espansione tumorale dopo sospensione della BRC. Laddove verificato, l'aumento delle dimensioni tumorali dopo sospensione della BRC è risultato efficacemente controllato dalla reintroduzione della terapia medica [9].

\section{Cabergolina}

Le prime evidenze pubblicate in letteratura nel corso degli anni Novanta hanno riportato un tasso di remissione variabile dal 13 al 31\% entro 6-12 mesi dopo sospensione della terapia con CAB [17-19]. Nel 2003 lo studio di Colao e colleghi [20] ha dimostrato che, dopo trattamento con CAB di durata mediana pari a 48 mesi, la sospensione della terapia farmacologica era seguita da remissione definitiva nel $76 \%$ dei pazienti con iperprolattinemia non tumorale, nel $70 \%$ di quelli con microprolattinoma e nel $64 \%$ di quelli con macroprolattinoma [20]. L'intervallo di tempo mediano tra la sospensione della $\mathrm{CAB}$ e il riscontro di recidiva di malattia era pari a 12 mesi nei pazienti con microprolattinoma, ovvero 18 mesi in quelli con iperprolattinemia non tumorale e con macroprolattinoma [20]. In particolare, il tasso di remissione a lungo termine risultava significativamente maggiore nei pazienti con iperprolattinemia non tumorale rispetto a quelli con microprolattinoma o macroprolattinoma, e nei pazienti senza evidenza radiologica di residuo tumorale all'atto della sospensione della $\mathrm{CAB}$ rispetto a quelli con evidenza radiologica di residuo tumorale [20], suggerendo pertanto un ruolo per la presenza di tessuto tumorale residuo e per le sue dimensioni quali predittori della remissione a lungo termine dopo interruzione della terapia farmacologica con CAB [20]. Pochi anni più tardi, le linee guida della Pituitary Society [21] hanno suggerito precisi criteri per la corretta gestione clinica della sospensione della terapia con DA nei pazienti con prolattinoma. Secondo tali raccomandazioni, la sospensione della terapia con DA può essere effettuata nei pazienti che: 1) abbiano ricevuto il trattamento con DA per almeno 2 anni; 2) abbiano raggiunto la completa normalizzazione dei valori di PRL; 3) abbiano mostrato una marcata riduzione ( $>50 \%$ ) delle dimensioni tumorali [21]. Inoltre, le linee guida della Pituitary Society hanno suggerito l'applicazione di un protocollo di graduale tapering del dosaggio dei DA, sottolineando l'importanza di una progressiva riduzione della dose di DA, evitandone la sospensione improvvisa, e di un attento follow-up dei pazienti dopo interruzione del trattamento [21]. Come mostrato nelle Tabelle 1 e 2, l'applicazione dei criteri di sospensione suggeriti dalle linee guida della Pituitary Society si è tradotta in un rate di remissione definitiva a lungo termine $(\geq 24$ mesi) pari al $49 \%$ dei pazienti [10-12, 15-24]. Inoltre, non sono emerse significative differenze nel tasso di remissione a lungo termine dopo sospensione della terapia con $\mathrm{CAB}$ tra pazienti con microprolattinoma $(45 \%)$ e quelli con macroprolattinoma (40\%) [10-12, 15-24]. Più recentemente, una meta-analisi degli studi che hanno valutato la remissione di iperprolattinemia dopo sospensione della terapia con DA ha riportato persistente normoprolattinemia nel $21 \%$ dei pazienti [25]. In particolare, il tasso di remissione di malattia risultava significativamente maggiore nei pazienti trattati con $\mathrm{CAB}$ rispetto a quelli trattati con $\mathrm{BRC}$, nei pazienti con iperprolattinemia non tumorale rispetto a quelli con microprolattinoma o macroprolattinoma, e nei pazienti trattati per più di due anni rispetto a quelli trattati per un periodo di tempo inferiore [25].

Gli studi, tuttavia, non hanno fornito un'univoca definizione di recidiva di iperprolattinemia dopo sospensione del trattamento con $\mathrm{CAB}$, dal momento che la definizione di recidiva è stata basata sul criterio puramente biochimico in tutti gli studi, ma anche sul criterio clinico di ricomparsa dei sintomi [23] o sul criterio radiologico della persistenza di tumore all'imaging ipofisario [26], senza peraltro indicare in maniera chiara come interpretare l'eventuale crescita del tumore dopo sospensione della terapia e senza definire in maniera precisa l'intervallo di tempo necessario alla rivalutazione dei pazienti per la diagnosi di recidiva di malattia. 
Tabella 1 Prevalenza della remissione di iperprolattinemia dopo sospensione della terapia con DA negli studi pubblicati prima delle raccomandazioni della Pituitary Society del 2006 [21]. BRC, bromocriptina; $C A B$, cabergolina

\begin{tabular}{|c|c|c|c|c|c|}
\hline Autore, anno & Pazienti (nr) & Remissione $\%$ & Terapia & Durata (mesi) & Follow-up (mesi) \\
\hline Johnston, 1984 & 15 & 7 & $\mathrm{BRC}$ & 42 & 12 \\
\hline Zarate, 1983 & 16 & 37 & $\mathrm{BRC}$ & 24 & 24 \\
\hline Moriondo, 1985 & 36 & 22 & $\mathrm{BRC}$ & 24 & 30 \\
\hline Wang, 1987 & 24 & 21 & $\mathrm{BRC}$ & 24 & $12-48$ \\
\hline Van't Verlaat, 1991 & 12 & 9 & $\mathrm{BRC}$ & $36-84$ & 12 \\
\hline Ferrari, 1992 & 127 & 31 & $\mathrm{CAB}$ & $40-84$ & 12 \\
\hline Muratori, 1997 & 26 & 19 & $\mathrm{CAB}$ & 12 & $38-60$ \\
\hline Cannavò, 1999 & 27 & 18 & $\mathrm{CAB}$ & 24 & 12 \\
\hline Passos, 2002 & 131 & 20 & $\mathrm{BRC}$ & 48 & 42 \\
\hline Colao, 2003 & 175 & 67 & $\mathrm{CAB}$ & 48 & $24-60$ \\
\hline Biswas, 2005 & 89 & 31 & $\mathrm{BRC}$ e $\mathrm{CAB}$ & 37 & 12 \\
\hline TOTALE & 678 & 35 & - & 42 (mediana) & 24 (mediana) \\
\hline
\end{tabular}

Tabella 2 Prevalenza della remissione di iperprolattinemia dopo sospensione della terapia con DA negli studi pubblicati dopo le raccomandazioni della Pituitary Society del 2006 [21]. BRC, bromocriptina; $C A B$, cabergolina

\begin{tabular}{llllll}
\hline Autore, anno & Pazienti (nr) & Remissione $\%$ & Terapia & Durata (mesi) & Follow-up (mesi) \\
\hline Colao, 2007 & 194 & 42 & CAB & $36-45$ & 48 \\
Kharlip, 2009 & 46 & 46 & CAB & 52 & 52 \\
Barber, 2011 & 60 & 29 & BRC e CAB & 90 & $3-36$ \\
Anagnostis, 2012 & 26 & 58 & BRC e CAB & 79 & 24 \\
TOTALE & 326 & 49 & - & 65 (mediana) & $36($ mediana) \\
\hline
\end{tabular}

\section{Predittori di remissione dopo sospensione della terapia}

Numerosi studi hanno valutato il ruolo di alcuni possibili predittori di remissione a lungo termine dopo sospensione della terapia con DA, tra cui la presenza di residuo tumorale ancora visibile all'imaging e le sue dimensioni [20], i valori di prolattina alla diagnosi [23] o al momento della sospensione del trattamento [22], la durata della terapia farmacologica e l'uso di CAB rispetto ad altri DA [25]. Per altri fattori, quali l'età, il sesso, le dimensioni del tumore alla diagnosi, la dose di DA utilizzata, la soppressione percentuale dei valori di prolattina, la presenza di concomitante ipopituitarismo alla diagnosi, la concomitante terapia con estroprogestinici e il precedente ricorso ad altre terapie quali l'intervento neurochirurgico e la radioterapia, non è stato documentato nessun ruolo prognostico. Nel 2007, uno studio di Colao e collaboratori [27] ha dimostrato che la prevalenza di remissione dopo sospensione del trattamento con $\mathrm{CAB}$ risultava significativamente maggiore nei pazienti che all'atto della sospensione avessero raggiunto valori di PRL $<162$ $\mathrm{mU} / \mathrm{L}$ associati a un residuo tumorale di diametro massimo $<3 \mathrm{~mm}$, rispetto ai pazienti che presentavano il solo crite- rio biochimico, il solo criterio radiologico o nessuno dei due [27].

\section{Cosa fare dopo un tentativo di sospensione fallimentare?}

Nei pazienti in cui la sospensione del trattamento con DA sia seguita da una recidiva di malattia, è suggerito di riprendere la terapia con i DA [1,2]. Tuttavia, la reintroduzione del trattamento non è da considerarsi definitiva, in quanto nei pazienti in cui siano rispettati i criteri suggeriti dalle linee guida della Pituitary Society del 2006 dopo due ulteriori anni di trattamento è possibile effettuare un secondo tentativo di sospensione, che è stato dimostrato essere efficace nel determinare persistente normoprolattinemia in circa un terzo dei casi $[28,29]$.

\section{Conclusioni}

In alcuni selezionati casi di pazienti con prolattinoma, la terapia con DA non è life-long. Infatti, i pazienti che raggiungono normali valori di prolattina e che mostrano una signifi- 
cativa riduzione delle dimensioni tumorali, fino alla non ulteriore visibilità del tumore all'imaging ipofisario, dopo terapia con DA per almeno due anni possono essere sottoposti a un protocollo di sospensione del trattamento farmacologico. La persistenza di normoprolattinemia dopo sospensione è altamente variabile a seconda del DA utilizzato e della durata della terapia stessa. Tuttavia, nei pazienti in cui sia verificata recidiva di malattia dopo un primo tentativo di sospensione dei DA, è suggerito di effettuare un secondo ciclo di trattamento con DA di ulteriori due anni e, quindi, proporre una seconda sospensione della terapia se siano rispettati i criteri per l'interruzione della stessa.

Funding Note Open access funding provided by Università degli Studi di Napoli Federico II within the CRUI-CARE Agreement.

Conflitto di interesse Gli autori Renata S. Auriemma, Roberta Scairati, Guendalina del Vecchio e Annamaria Colao dichiarano di non avere conflitti di interesse. Renata S. Auriemma fa parte del Comitato di redazione.

Consenso informato Lo studio presentato in questo articolo non ha richiesto sperimentazione umana.

Studi sugli animali Gli autori di questo articolo non hanno eseguito studi sugli animali.

Nota della casa editrice Springer Nature rimane neutrale in riguardo alle rivendicazioni giurisdizionali nelle mappe pubblicate e nelle affiliazioni istituzionali.

Open Access This article is licensed under a Creative Commons Attribution 4.0 International License, which permits use, sharing, adaptation, distribution and reproduction in any medium or format, as long as you give appropriate credit to the original author(s) and the source, provide a link to the Creative Commons licence, and indicate if changes were made. The images or other third party material in this article are included in the article's Creative Commons licence, unless indicated otherwise in a credit line to the material. If material is not included in the article's Creative Commons licence and your intended use is not permitted by statutory regulation or exceeds the permitted use, you will need to obtain permission directly from the copyright holder. To view a copy of this licence, visit http://creativecommons.org/licenses/by/4.0/.

\section{Bibliografia}

1. Melmed S, Casanueva FF, Hoffman AR, et al (Endocrine Society) (2011) Diagnosis and treatment of hyperprolactinemia: an Endocrine Society clinical practice guideline. J Clin Endocrinol Metab 96(2):273-288

2. Gillam MP, Molitch ME, Lombardi G, Colao A (2006) Advances in the treatment of prolactinomas. Endocr Rev 27(5):485-534

3. Auriemma RS, Grasso LF, Pivonello R, Colao A (2016) The safety of treatments for prolactinomas. Expert Opin Drug Saf 15(4):503-512

4. Tindall GT, Kovacs K, Horvath E, Thorner MO (1982) Human prolactin-producing adenomas and bromocriptine: a histological, immunocytochemical, ultrastructural, and morphometric study. J Clin Endocrinol Metab 55(6):1178-1183
5. Bassetti M, Spada A, Pezzo G, Giannattasio G (1984) Bromocriptine treatment reduces the cell size in human macroprolactinomas: a morphometric study. J Clin Endocrinol Metab 58(2):268-273

6. Gen M, Uozumi T, Ohta M et al (1984) Necrotic changes in prolactinomas after long term administration of bromocriptine. J Clin Endocrinol Metab 59(3):463-470

7. Landolt AM, Osterwalder V (1984) Perivascular fibrosis in prolactinomas: is it increased by bromocriptine? J Clin Endocrinol Metab 58(6):1179-1183

8. Orrego JJ, Chandler WF, Barkan AL (2000) Rapid re-expansion of a macroprolactinoma after early discontinuation of bromocriptine. Pituitary 3(3):189-192

9. Thorner MO, Perryman RL, Rogol AD et al (1981) Rapid changes of prolactinoma volume after withdrawal and reinstitution of bromocriptine. J Clin Endocrinol Metab 53(3):480-483

10. Passos VQ, Souza JJ, Musolino NR, Bronstein MD (2002) Long-term follow-up of prolactinomas: normoprolactinemia after bromocriptine withdrawal. J Clin Endocrinol Metab 87(8):3578-3582

11. Johnston DG, Hall K, Kendall-Taylor P et al (1984) Effect of dopamine agonist withdrawal after long-term therapy in prolactinomas. Studies with high-definition computerised tomography. Lancet 2(8396):187-192

12. Moriondo P, Travaglini P, Nissim M et al (1985) Bromocriptine treatment of microprolactinomas: evidence of stable prolactin decrease after drug withdrawal. J Clin Endocrinol Metab 60(4):764-772

13. Wang C, Lam KS, Ma JT et al (1987) Long-term treatment of hyperprolactinaemia with bromocriptine: effect of drug withdrawal. Clin Endocrinol (Oxf) 27(3):363-371

14. Winkelmann W, Allolio B, Heesen D et al (1988) Impaired regulation of prolactin in patients with persisting normoprolactinaemia after withdrawal of long-term therapy with dopamine agonists. In: Landolt AM, Heitz PU, Zapf J et al (eds) Advances in pituitary adenoma research. Pergamon Press, Oxford, p 317

15. van't Verlaat JW, Croughs RJ (1991) Withdrawal of bromocriptine after long-term therapy for macroprolactinomas; effect on plasma prolactin and tumour size. Clin Endocrinol (Oxf) 34(3):175-178

16. Zárate A, Canales ES, Cano C, Pilonieta CJ (1983) Followup of patients with prolactinomas after discontinuation of longterm therapy with bromocriptine. Acta Endocrinol (Copenh) 104(2):139-142

17. Ferrari C, Paracchi A, Mattei AM et al (1992) Cabergoline in the long-term therapy of hyperprolactinemic disorders. Acta Endocrinol (Copenh) 126(6):489-494

18. Muratori M, Arosio M, Gambino G et al (1997) Use of cabergoline in the long-term treatment of hyperprolactinemic and acromegalic patients. J Endocrinol Invest 20(9):537-546

19. Cannavò S, Curtò L, Squadrito S et al (1999) Cabergoline: a firstchoice treatment in patients with previously untreated prolactinsecreting pituitary adenoma. J Endocrinol Invest 22(5):354-359

20. Colao A, Di Sarno A, Cappabianca P et al (2003) Withdrawal of long-term cabergoline therapy for tumoral and nontumoral hyperprolactinemia. N Engl J Med 349(21):2023-2033

21. Casanueva FF, Molitch ME, Schlechte JA et al (2006) Guidelines of the Pituitary Society for the diagnosis and management of prolactinomas. Clin Endocrinol (Oxf) 65(2):265-273

22. Kharlip J, Salvatori R, Yenokyan G, Wand GS (2009) Recurrence of hyperprolactinemia after withdrawal of long-term cabergoline therapy. J Clin Endocrinol Metab 94(7):2428-2436

23. Biswas M, Smith J, Jadon D et al (2005) Long-term remission following withdrawal of dopamine agonist therapy in subjects with microprolactinomas. Clin Endocrinol (Oxf) 63(1):26-31

24. Barber TM, Kenkre J, Garnett C et al (2011) Recurrence of hyperprolactinaemia following discontinuation of dopamine agonist therapy in patients with prolactinoma occurs commonly especially in macroprolactinoma. Clin Endocrinol (Oxf) 75(6):819-824 
25. Dekkers OM, Lagro J, Burman P et al (2010) Recurrence of hyperprolactinemia after withdrawal of dopamine agonists: systematic review and meta-analysis. J Clin Endocrinol Metab 95(1):43-51

26. Anagnostis P, Adamidou F, Polyzos SA et al (2012) Long term follow-up of patients with prolactinomas and outcome of dopamine agonist withdrawal: a single center experience. Pituitary 15(1):25-29

27. Colao A, Di Sarno A, Guerra E et al (2007) Predictors of remission of hyperprolactinaemia after long-term withdrawal of cabergoline therapy. Clin Endocrinol (Oxf) 67(3):426-433
28. Kwancharoen R, Auriemma RS, Yenokyan G et al (2014) Second attempt to withdraw cabergoline in prolactinomas: a pilot study. Pituitary 17(5):451-456

29. Vilar L, Albuquerque JL, Gadelha PS et al (2015) Second attempt of cabergoline withdrawal in patients with prolactinomas after a failed first attempt: is it worthwhile? Front Endocrinol (Lausanne) 6:11 